\title{
Temperature profiling of diode lasers and laser bars by means of infrared thermography
}

\author{
by A. Kozłowska*, A. Maląg*, M. Teodorczyk*, E. Dąbrowska* and D. Podniesiński^* \\ ^Institute of Electronic Materials Technology, 133 Wólczyńska St., 01-919 Warsaw, Poland, \\ anna.kozlowska@itme.edu.pl \\ **Military University of Technology, Institute of Optoelectronics, 2 Kaliskiego St., 00-908 Warsaw, Poland.
}

\begin{abstract}
Thermal characterization of individual diode lasers and laser bars by means of infrared thermography is performed. Different heat sink solutions suitable for high power devices are analyzed. Temperature profiling results for individual diode lasers mounted on copper blocks and in modified TO-3 packages are presented. For laser bars active cooling with different constructions of micro-channel heat sinks is employed. NOVEC 7000 series fluids and alternatively deionized water is used as a cooling medium. Methodological approach to thermographic characterization of high-power diode lasers and laser bars is presented.
\end{abstract}

\section{Introduction}

High power diode lasers and laser bars require very efficient thermal systems that enable to remove the heat fluxes resulting from the high power operation. Whereas for individual diode lasers a conductive cooling is in the most cases an appropriate solution, for laser bars the improved thermal management systems including micro-channel heat sinks are analyzed and developed [1-4]. Inspection of thermal profiles of operating devices is here of paramount importance and infrared thermography offers interesting characterization possibilities $[5,6]$. In present work, infrared thermography is used for temperature profiling of diode lasers and laser bars mounted on different heat sinks. For individual diode lasers the thermal behaviour when mounted on laboratory Cu-blocks and in modified TO-3 packages will be compared. For laser bars the results of temperature profiling of devices mounted on prototype micro-channel heat sinks will be presented.

\section{Experiment and exemplary results}

Thermal properties of diode lasers emitting at $808 \mathrm{~nm}$ are analyzed. Individual diode lasers are based on Double Barrier Separate Confinement Heterostructure (DB SCH) [7]. Wide-stripe $(w=0.1 \mathrm{~mm})$, gain guided laser structures are fabricated using $\mathrm{He}^{+}$implantation for stripe definition. TiPt and AuGe metallizations are used as $\mathrm{p}$ - and $\mathrm{n}$ contacts, respectively. Lasers of cavity length $L=2 \mathrm{~mm}$ are formed by cleaving and subsequent HR/LR (high reflectivity/low reflectivity) facet coating. The devices are mounted using indium on copper blocks and in TO-3 packages. Commercial laser arrays are mounted on novel construction copper heat sinks. The heat sinks contain micro-channels with miniature pipes inside. NOVEC 7000 series fluids (7000 and $7100,3 \mathrm{M}$ ) or deionized water is used as a coolant.

The experiments are carried out using the THERMOSENOSORIK 640 SM camera equipped with a $640 \times 512$ pixel InSb-detector (wavelength range 1,1 - 5,3 micron). An IR-micro-objective ( $f / 8$ ) with magnification of $10 \times$ and IR-lens with a focal length $f=28 \mathrm{~mm}$ are used. The laser diode radiation coming from interband quantum well transitions is rejected using a Si-wafer as a filter. This allows to avoid additional heating of the lens and the detector during laser operation. The calibration characteristics are measured for each batch of the devices characterized. The temperature of non-operating diode laser / heat sink assembly is stabilized by TEC, operating in the heating/cooling mode. For each temperature setting a thermal image of the device is captured with the camera. Exemplary calibration images for an individual diode laser are shown in Fig. 1a-c. The calibration data is approximated by third-order polynomial (Fig. 1d).

Exemplary results of thermographic characterization of individual diode laser mounted in a modified TO-3 package are shown in Fig. 2. A thermal image observed from front facet of laser diode operating at $\mathrm{I}=1.4 \mathrm{~A}$ and horizontal temperature profiles for three current levels are shown in Fig. $2 a$ and $2 b$, respectively.

\section{Conclusions}

Infrared thermography is a powerful tool for thermal inspection of high-power diode lasers. Measurements of temperature profiles allow to evaluate the heat removal efficiency of the heat sink. This feature is especially helpful by the development of novel heat sinks for high power diode lasers.

\section{Acknowledgements}

This work was supported by National Centre for Research and Development under the research projects N R02 000406 and N R02 001306. 


\section{REFERENCES}

[1] Tomm J.W., Jimenez J. Ed., „Quantum-Well Laser Array Packaging”, McGraw-Hill, Nanoscience and Technology Series, 2007.

[2] Vetrovec J., "Improved cooling for high-power laser diodes", Proc. SPIE, vol. 6876, pp. 687603-1-7, 2008.

[3] Huddle J.J., L.C. Chow, S. Lei, A. Marcos, D.P. Rini, S.J. Lindauer, M. Bass, P.J. Delfyett, "Thermal management of diode laser arrays", Sixteenth IEEE SEMI-THERM Symposium Proceedings, pp. 154-160, 2000.

[4] Dix J., Jokar A., Martinsen R., "Enhanced microchannel cooling for high-power semiconductor diode lasers", Proc. SPIE, vol. 6876, pp. 687606-1-10, 2008.

[5] Kozłowska A., "Infrared imaging of semiconductor lasers", Semicond. Science \& Technol., vol. 22, pp. R27-R40, 2007.

[6] Kozłowska A., Ziegler M., Tomm J.W., Sarzała R.P., Nakwaski W., "Thermal imaging of actively cooled highpower laser bars", Proceedings of Mixed Design of Integrated Circuits and Systems MIXDES 2007, 21-23 June, Ciechocinek, 2007.

[7] Maląg A., Jasik A., Teodorczyk M., Jagoda A., Kozłowska A., "High-power low vertical beam divergence 800-nmband double-barrier-SCH GaAsP-(AIGa)As laser diodes", IEEE Photon. Tech. Lett., vol. 18, 1582-4, 2006.

a)

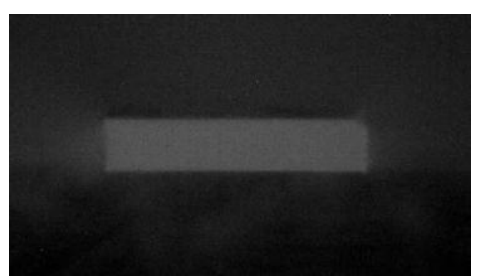

b)

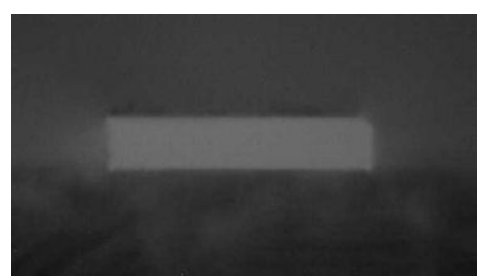

c)

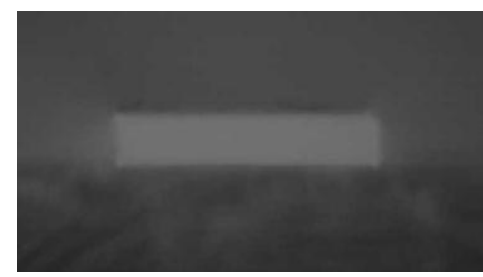

d)

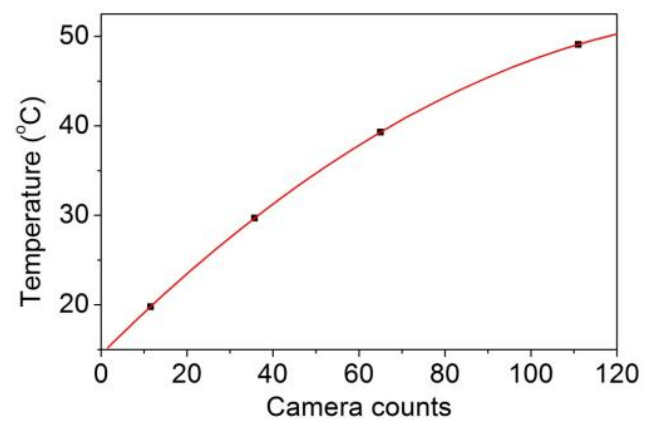

Fig. 1. Exemplary calibration images captured at $30{ }^{\circ} \mathrm{C}$ (a), $40{ }^{\circ} \mathrm{C}$ (b) and $50{ }^{\circ} \mathrm{C}$ (c) of non-operating diode laser and calibration data for the active region area $(d)$.

a)

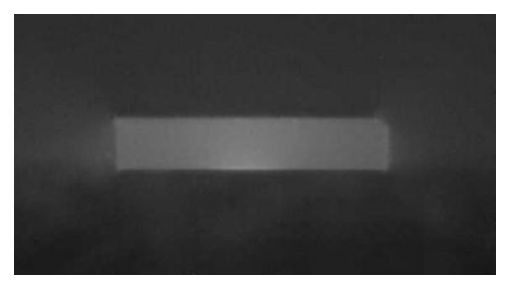

b)

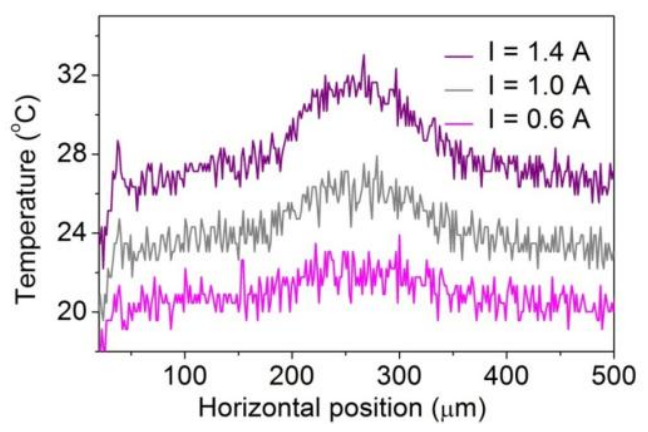

Fig. 2. Front view thermal image of an exemplary diode laser (a) and corresponding horizontal temperature profiles for three current levels (b). 